\title{
Less common forms of posterior canal benign paroxysmal positional vertigo
}

\author{
Forme meno comuni di vertigine parossistica benigna da posizionamento \\ Luigi Califano, Salvatore Mazzone, Francesca Salafia, Maria Grazia Melillo, Giuseppe Manna \\ San Pio Hospital, Department of Audiology and Phoniatrics, Benevento, Italy
}

\begin{abstract}
SUMMARY
The most common form of posterior canal benign paroxysmal positional vertigo (BPPV) is characterised by positional, paroxysmal, upbeat nystagmus, with a torsional component beating towards the downward ear. Rarer variants have been reported, putatively due to either the position of otoconia in the canal or anatomic variations of the semicircular canals. The most frequent less common form is apogeotropic posterior canal BPPV, in which the positional nystagmus is downbeat and torsional apogeotropic. In this form, the main problems are differential diagnosis with central mimics of BPPV and identification of the affected canal and its side. The authors propose a new subtype of apogeotropic posterior canal BPPV hypothesising the localization of otoconia in the ampullary tract of the affected canal, which might explain the modality of the conversion in typical posterior canal BPPV which they observed in some of these cases. If nystagmus of the less common forms of posterior canal BPPV is explicable through purely peripheral mechanisms, these forms should no longer be defined as "atypical" but, in a less demanding way, as "less common" variants, which must be recognised by any expert otoneurologist.
\end{abstract}

KEY WORDS: posterior canal BPPV, canalolithiasis, apogeotropic posterior canal BPPV, posterior canal BPPV less common variants

\section{RIASSUNTO}

La forma più comune di vertigine parossistica posizionale benigna (VPPB) del canale posteriore è caratterizzata da nistagmo posizionale, parossistico, verticale in alto, con componente torsionale verso l'orecchio posizionato più in basso. Varianti meno comuni sono presumibilmente dovute alla posizione degli otoliti nel canale o a variazioni anatomiche dei canali semicircolari. La forma rara più frequente è quella apogeotropa, in cui il nistagmo posizionale batte verso il basso e la componente torsionale batte in opposizione all'orecchio malato. In questa forma, i problemi principali sono la diagnosi differenziale con le forme centrali di vertigine posizionale e l'identificazione del canale e lato interessati.Gli autori propongono un nuovo sottotipo di VPPB apogeotropa del canale posteriore, ipotizzando la localizzazione degli otoconi nel tratto ampollare del canale interessato, il che potrebbe spiegare la modalità della conversione in forma tipica del canale posteriore osservata in alcuni di questi casi. Se il nistagmo delle forme meno comuni di VPPB del canale posteriore è spiegabile attraverso meccanismi periferici, queste forme non dovrebbero più essere definite come "atipiche" ma, in modo meno impegnativo, come varianti "meno comuni” la cui conoscenza è richiesta all'Otoneurologo esperto.

PAROLE CHIAVE: VPPB da canalolitiasi posteriore, canalolitiasi, VPPB da canalolitiasi posteriore apogeotropa, varianti meno comuni di VPPB da canalolitiasi posteriore

\section{Introduction}

The topographical classification of benign paroxysmal positional vertigo (BP$\mathrm{PV}$ ) is based on the position of otoconia in the semicircular canals, which can
Received: January 12, 2021

Accepted: February 27, 2021

Correspondence

Luigi Califano

San Pio Hospital, Department of Audiology and Phoniatrics, Benevento, Italy

via A. Lepore 1, 82100 Benevento, Italy

Tel. +39 0824 57407. Fax +39082457430

E-mail: luigi.califano@tin.it

Funding

None.

Conflict of interest

The Authors declare no conflict of interest.

How to cite this article: Califano L, Mazzone S, Salafia F, et al. Less common forms of posterior canal benign paroxysmal positional vertigo. Acta Otorhinolaryngol Ital 2021;41:255-262. https:// doi.org/10.14639/0392-100X-N1032

(C) Società Italiana di Otorinolaringoiatria e Chirurgia Cervico-Facciale

\section{(c) (1) $(2)$}

This is an open access article distributed in accordance with the CC-BY-NC-ND (Creative Commons Attribution-NonCommercial-NoDerivatives 4.0 International) license. The article can be used by giving appropriate credit and mentioning the license, but only for non-commercial purposes and only in the original version. For further information: https:// creativecommons.org/licenses/by-nc-nd/4.0/deed.en 
hypothetically be inferred through the oculomotor responses observed during positioning tests and determined by the endolymphatic flow induced in the affected semicircular canal. According to Ewald's laws, in the vertical canals, ampullofugal flow is excitatory and ampullopetal flow is inhibitory, whereas the opposite occurs in the lateral canals. Ampullary responses to endolymphatic flow cause the activation/inhibition of the extra-ocular muscles, which are anatomically connected to the vestibular system through a neural circuitry that mediates the vestibulo-oculomotor reflex. Thus, the coordinated responses of extra-ocular muscles evoke nystagmus in predictable ways.

In the typical form of posterior canal BPPV (TPC), positioning test evokes paroxysmal nystagmus with fast phases directed towards the upper ocular pole for the vertical component and towards the downward ear for the torsional component ("geotropic" form) ${ }^{1}$ (Fig. 1).

Less common clinical variants of posterior canal BPPV have also been reported: 1. "Reversed posterior canal BPPV", 2,3 characterised by downbeat and torsional nystagmus beating towards the inferior ocular pole and the upward ear (apogeotropic nystagmus); 2. "Short arm canalolithiasis" in which otoconia are in the utricular arm of the posterior ampulla $^{4} ; 3$. Subjective BPPV and Type 2 BPPV ${ }^{5,6}$ : during the positioning tests, some patients experience only subjective symptomatology without positional nystagmus (subjective BPPV). In returning to the sitting position, they feel a short and intense dizzy sensation accompanied by a sense of retropulsion, up to almost falling in some cases; 4 . Sitting Up Vertigo ${ }^{7}$, in which positional vertigo and positioning nystagmus typical of TPC is evoked when the patient

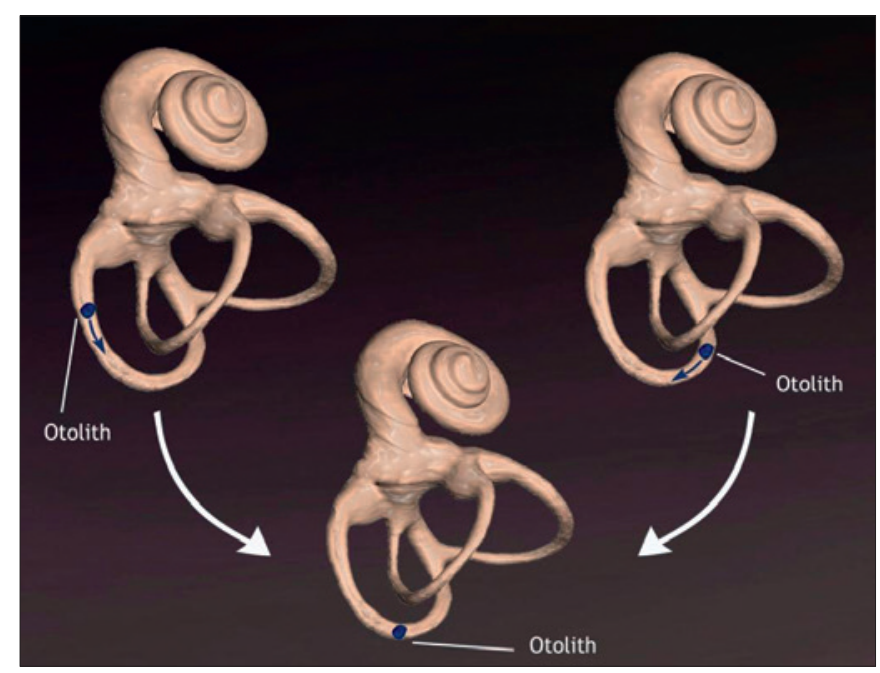

Figure 1. During the positioning test otoconia (blue circles) move ampullophugally in typical posterior canal BPPV (on the left), move ampullopetally in apogeotropic posterior canal BPPV (on the right). sits up from the Dix-Hallpike position on the affected side; 5. Posterior canal heavy cupula ${ }^{8}$ caused either by otoconia attached to the cupula -cupulolithiasis- or by a transitory modified density ratio between the cupula and the surrounding endolymph. 6. Posterior canal light cupula ${ }^{9-11}$, characterised by a cupula with a specific gravity lower than the endolymph. 7. Yetiser's variant ${ }^{12}$, characterised by a biphasic paroxysmal nystagmus, whose first phase presents a torsional component reversed respect to the side of stimulation.

Our retrospective cohort study analysed the frequency of the so-called atypical forms of posterior canal BPPV, their response to physical therapy and their evolution. Data from the most frequent of them, the apogeotropic form, the only one which was susceptible to numerical analysis, were examined. We also considered its modeling and the modalities of its possible conversion in TPC, finally hypothesising a new possible subtype of this form. We will also provide critical analysis of the rarest forms of posterior canal BPPV.

\section{Patients and methods}

From January 2019 to February 2020 we observed 630 cases of acute BPPV: 466 TPC, diagnosed and treated according to current diagnostic and therapeutic guidelines ${ }^{1}$, 25 apogeotropic variant of posterior BPPV (APC) and 4 anterior canal BPPV (AC), for a total of 29 cases of positional downbeat nystagmus (PDN), 5 cases ascribable to Scocco's variant, 2 cases ascribable to Yetiser's variant and 128 lateral canal BPPV. We used criteria set out in a previous paper ${ }^{13}$ to distinguish between AC and APC, maintaining their classification in three grades (Tabs. I, II). In the same period, we observed 34 central PDNs that will not be the object of our presentation. Another 25 cases were ascribed to either "subjective BPPV" (10 cases) or "trunk retropulsion in sitting up without observable nystagmus" (15 cases). Patients underwent otomicroscopy, pure tone audiometry and infrared videonystagmoscopy. Video head impulse test (v-HIT) was performed in 205 patients. The diagnosis of vertical canal BPPV was based on the DixHallpike test and head hanging positioning. After informed consent, 466 TPC cases were treated through quick liberatory rotation manoeuver (QLR) ${ }^{14}$, Epley manoeuver or Semont manoeuver; 25 APC cases were treated either through QLR or through Vannucchi manoeuver ${ }^{15}$. Brain MRI was planned in APC and AC “possible” forms. Scocco's variant cases were treated through QLR, Epley manoeuver and Brandt-Daroff protocol. Yetiser variant cases were treated through QLR; "Subjective BPPV" cases were treated and resolved through a liberatory manoeuver - QLR, Epley ma- 
Table I. Anterior canal BPPV.

\section{a) Definite AC BPPV}

Presence of a positional vertical down-beating paroxysmal nystagmus evoked through the straight head-hanging positioning and sometimes through the DixHallpike test

In such positions, possible presence of a slight clockwise torsional component for the left AC or of a slight counterclockwise torsional component for the right $\mathrm{AC}$

Canal conversion in typical posterior canal BPPV during or immediately after (no more than two days) the therapeutic manoeuvre, characterised by a vertical up-beating nystagmus, clockwise for the left canal and counterclockwise for the right canal
\end{abstract}

\title{
b) Probable AC BPPV
}

As reported in la, but with a direct resolution of disease without canalar conversion in typical posterior canal BPPV

\section{c) Possible AC BPPV}

Persistence of symptoms after 5 cycles of therapeutic manoeuvres

MRI does not show any neurological disease as a presumed cause of the nystagmus

or

Patient lost to follow-up before the resolution of the disease

AC: anterior canal; BPPV: benign paroxysmal positional vertigo; MRl: magnetic resonance imaging.

Table II. Apogeotropic posterior canal BPPV.
a) Definite APC BPPV
Presence of torsional apogeotropic nystagmus, evoked through the Dix-Hallpike test and sometimes through the straight head hanging positioning
Presence of a vertical down-beating component in the same positioning tests
Canalar conversion in typical posterior canal BPPV during or immediately after (no more than two days) the therapeutic manoeuvre, or suddenly during the prolonged maintenance of one of the head hanging positions (straight head hanging; "deep" Dix-Hallpike position) or during the maintenance of leaning position in sitting position

\section{b) Probable APC BPPV}

As reported in lla, but with a direct resolution of disease without canalar conversion in typical posterior canal BPPV

\author{
c) Possible APC BPPV \\ Persistence of symptoms after 5 cycles of therapeutic manoeuvres \\ $\mathrm{MRI}$ does not show any neurological disease as a presumed cause of the nystagmus \\ or
}

Patient lost to follow-up before the resolution of the disease

APC: apogeotropic posterior canal; BPPV: benign paroxysmal positional vertigo; MRl: magnetic resonance imaging.

noeuver or Semont manoeuver - performed starting from the side of the referred symptoms; "Trunk retropulsion in sitting up without observable nystagmus" cases were treated through a home protocol, derived from the original proposal by Büki et al. ${ }^{5}$, based on the repetition of DixHallpike positioning followed by the return in the sitting position for 10 consecutive times, three times a day for seven consecutive days.

Our analysis focused mainly on the diagnosis, treatment and modelling of APC cases, the only "atypical" group susceptible to numerical analysis. Statistical analysis was performed with descriptive statistics and confidence interval of a mean, and unpaired T-test, with significance level set at $0.05,95 \%$ confidence interval, using the Graph pad Quick Calcs software.

All procedures were in accordance with the ethical standards of the Ethics Review Board and with the 1964 Helsinki declaration and its later amendments.

\section{Results}

BPPV cases accounted for $24.8 \%$ of all vertiginous and dizzy patients we observed in the same period, representing the first cause of vertigo in our series. In our geographic area, BPPV incidence was 132/100,000 per year.

APC represented $5.02 \%$ of the overall acute posterior canal BPPV cases ( 25 out of 498 cases), much more frequent than AC ( 4 cases) (ratio APC/AC: 6.25/1). In 21/29 cases of peripheral PDN, positional vertigo caused by movements in the pitch plane was the main symptom. Four patients reported only sub-continuous dizziness and intolerance to movements. In the APC group, the mean age was $58.6 \pm 10.34$, the female/male ratio $=1.32$, which was not significantly different from the overall posterior canal BPPV population (mean age 59.6 \pm 7.45 , female/male ratio $=1.36$ ).

463/466 cases of TPC were resolved through QLR, or Epley manoeuver, or Semont manoeuver; the mean number of liberatory manoeuvers needed to cure was $2.07 \pm 0.79$ 
(range 1-5), without significant differences between the three manoeuvers.

12 APC cases were treated through QLR, 6 cases through Vannucchi manoeuver and 1 case through both QLR and Vannucchi manoeuver; 6 cases were converted in TPC through the maintenance of a hyperextension position of the head, and then treated through QLR (see later). The direct resolution of APC (one step therapy, "probable" grade) was obtained in 10/25 patients (40\%), in 7 cases through QLR, in 3 cases through Vannucchi manoeuver. The mean number of QLR in the one-step therapy was $2.71 \pm 0.95$, which was significantly higher than in TPC $(p=0.03)$. APC converted in TPC ("definite grade") in 14/25 cases (56\%): in 5 cases through QLR, in 3 cases through Vannucchi manoeuver, in 6 cases suddenly and intensely during the prolonged maintenance of a forced head hyperextension position: head hanging position in 2 cases, Dix-Hallpike position on the affected side in 3 cases, leaning position in 1 case, sometimes without any dynamic movement, in a time ranging between 45 seconds and 5 minutes, and sometimes after a facilitating technique, i.e. a rapid bowing of the head followed by hyperextension in "deep" Dix-Hallpike or head hanging position. The mean number of QLR and Vannucchi manoeuvers necessary to convert APC into TPC was $2.26 \pm 0.81$. One case (4\%) with negative brain MRI failed the treatment ("possible" grade).

All APC cases converted in TPC were then cured through QLR (two-step therapy), by a mean number of manoeuvers of $2.46 \pm 0.88$, which was slightly significantly higher than in TPC cases $(\mathrm{p}=0.05)$.

Three AC cases were solved through Yacovino manoeuver, 1 case was not resolved, and treated through Brandt-Daroff home protocol.

In a 3-17 month follow-up, last evaluation in May 2020, 2 patients of the peripheral PDN group relapsed in ipsilateral TPC, 1 in ipsilateral APC and 1 in ipsilateral geotropic lateral canal BPPV. No patient showed signs and/or symptoms of neurological disease.

All the cases of both "subjective BPPV" and "trunk retropulsion in sitting up without observable nystagmus" were characterised by a significant delay in diagnosis and treatment, in all cases more than 40 days from the onset of acute and typical symptoms which have progressively disappeared. "Subjective BPPV" cases were resolved through $1.40 \pm 0.52$ therapeutic manoeuvers; "trunk retropulsion in sitting up without observable nystagmus" cases were resolved through one cycle of the above described home protocol.

\section{Discussion}

Some forms of presumed posterior canal BPPV are characterised by nystagmus that is different from the TPC nys- tagmus. In the past, such forms were "tout-court" defined as atypical. Epley ${ }^{16}$ considered atypical all forms of positional vertigo whose evoked nystagmus did not respect the usual patterns. Rarer variants of posterior canal BPPV do exist and are sustained by various clinical observations ${ }^{2-13}$. They are explained either by anomalous localisation of otoconia in different tracts of the affected posterior canal or by anatomical anomalies or structural changes of semicircular canals, such as stenosis and fibrosis, as high definition MRI and micro-CT three-dimensional reconstruction have suggested ${ }^{17,18}$. In the above-mentioned situations, movements of otoconia causing endolymphatic flows different from the typical ampullofugal current occurring in TPC during positional tests are believed to occur: for example, ampullopetal flow in APC, or ampullofugal flow in sitting-up in Scocco's variant.

1. "Reversed posterior canal BPPV" is characterised by downbeat and torsional nystagmus beating towards the inferior ocular pole and the upward ear (apogeotropic nystagmus). This form is associated with otoconia displaced in the non-ampullary tract of the posterior canal ${ }^{2,3,13}$. During diagnostic tests, otoconia move away from the non-ampullary tract of the posterior canal and provoke an ampullopetal endolymphatic flow in the affected canal (Fig. 1). This causes the inhibitory response of the ampullary receptor that evokes both the vertical downbeat vector and the torsional component of the positional nystagmus. Vannucchi called this clinical scenario "apogeotropic posterior canal BPPV" (APC) ${ }^{3}$. In APC, nystagmus is usually evoked in both Dix-Hallpike positions and head hanging position. It seldom reverses in returning to the sitting position. Nystagmus is caused by the activation of the same ocular muscles activated in contralateral anterior canal BPPV (AC), ipsilateral superior rectus muscle and contralateral inferior obliquus, so the differentiation between the two forms is sometimes difficult ${ }^{3,13}$.

Büki ${ }^{6}$ provided another interesting explanation of at least some cases of PDN from posterior canalolithiasis. If detached otoconia enter the common crus or if the patient liberates himself by turning over in bed, or if otoconia detach when the patient is standing, otoconia remain or return into the utriculum. Here, they may be re-located in the pericupular short arm and fall on the posterior canal cupula which, in the upright position, is just below the utricular macula. The cupula of the posterior canal would adapt itself to the greater weight present on it, chronically deflecting itself downward. In these cases, in either the Dix-Hallpike position or the head hanging position, nystagmus may not be evoked. If evoked, it is downbeat, slight, and long-lasting. This is determined both by the spatial orientation of the cupula, which is now a graviceptive organ and by its upward displacement. 
The main problem when observing a PDN is its possible origin from a central, particularly cerebellar, disease ${ }^{19-21}$. In these cases, nystagmus is not provoked by otoconia in a semicircular canal but by impaired "central vestibular processing of canal and otolith in addition, to prolonged velocity storage in the cerebellar nodulus" ${ }^{20}$. In central PDN, further central ocular motor abnormalities are usually present. Other important criteria for the differential diagnosis are the conversion of the peripheral PDN in TPC and/or its resolution through a liberatory manoeuvre. In all the other cases, brain MRI is mandatory.

2. Oas ${ }^{4}$ hypothesised the "short arm canalolithiasis" in which otoconia are in the utricular arm of the posterior ampulla. After the paroxysmal phase of the positional nystagmus has ended, the short arm canalolithiasis provokes a slight and persistent ipsidirectional torsional nystagmus; differently, in the typical form - long arm canalolithiasis - a transient reversed torsional nystagmus happens. During the repositioning manoeuvre, instead, the short arm canalolithiasis would be characterised by the reversal of the nystagmus. The author proposed an "ad hoc" therapeutic protocol for such a form.

3. Subjective BPPV and Type 2 BPPV ${ }^{5,6}$. The authors observed 200 consecutive patients from 3 to 5 months after the onset of positioning vertigo, reporting a TPC in $19.5 \%$. During the positioning tests, $43 \%$ of patients experienced only subjective symptomatology without positional nystagmus (subjective BPPV). In returning to the sitting position, they felt a short and intense dizzy sensation accompanied by a sense of retropulsion, up to almost falling in some cases. In about $50 \%$ of these cases, patients reported abnormal trunk oscillations which were objectively quantified by an "ad hoc" posturography in the sitting position. The authors called this form "Type 2 BPPV", caused by the presence of free-floating otoconia in the utriculus near the posterior canal cupula. Otoconia produced neither significant cupular displacement nor nystagmus in the Dix-Hallpike positioning. When patients returned to the sitting position, otoconia floated in the short arm causing dizziness, sometimes with compliant of retropulsion. The authors proposed repetitive sits-up from the Dix-Hallpike position as liberatory manoeuvre.

4. Scocco et al. ${ }^{7}$ described positional vertigo and positioning nystagmus typical of TPC but evoked when the patient sits up from the Dix-Hallpike position on the affected side (Sitting Up Vertigo). They often observed this variant after a repositioning manoeuvre. Either a narrowing of the canal in the periampullary arm or large-sized debris block otoconia to move distally in the Dix-Hallpike positioning. When the patient returns quickly to the sitting position, during the movement, otoconia move inertially towards the cupula.
Afterwards, due to gravitational force, otoconia slide back ampullofugally towards the narrow tract evoking a "counter-positioning" nystagmus analogous in its characteristics to the TPC nystagmus usually evoked in the ipsilateral DixHallpike position. This variant is often resistant to repositioning manoeuvres: in fact, the authors always resolved their cases through Brandt-Daroff home protocol.

5. Yetiser ${ }^{12}$ described a single case of paroxysmal nystagmus evoked by the Dix-Hallpike test on the left side. Nystagmus was biphasic: after a short latency, its first phase was paroxysmal, upbeat and counterclockwise, immediately followed by a second phase, upbeat and clockwise, with lower intensity and longer duration. The author concluded that nystagmus would be caused by canalolithiasis of the non-ampullary arm of the left posterior canal. The second phase of the nystagmus would be due to the ampullofugal reflux of the endolymph after the initially evoked ampullopetal flow.

6. Posterior canal heavy cupula ${ }^{8}$. This condition would be caused either by otoconia attached to the cupula - cupulolithiasis - or by a transitory modified density ratio between the cupula and the surrounding endolymph. The second situation is typically observed in migraineurs with frequently recurrent spells of positional vertigo (migrainerelated positional vertigo). The density of the endolymph would be modified by metabolic changes due to migraine attacks, e.g, localised fluid diffusions from the labyrinth microcirculation to the endolymph. Posterior cupulolithiasis and heavy cupula from pericupular light endolymph are just slightly different from canalolithiasis in the features of the evoked positional nystagmus: quick phase direction is similarly geotropic, upbeat and rotatory towards the downmost ear, but the nystagmus has short or no latency, is less paroxysmal, longer-lasting and less fatigable. Liberatory manoeuvres are not effective in heavy cupula from light endolymph; instead, physical therapy works better in cupulolithiasis, and mastoid vibration can help to detach otoconia from the cupula. Because the evoked nystagmus is the same as TPC, albeit longer-lasting and less paroxysmal, in our analysis we will not consider this form as "atypical". 7. Posterior canal light cupula ${ }^{9-11}$. "Light cupula" is characterised by a cupula with a specific gravity lower than the endolymph. It is described in the first phase of post alcohol nystagmus (positional direction-changing nystagmus). Ichimura et al. ${ }^{16}$ reported a case of a 16 -year-old boy with rotatory positional vertigo visited on the next day after symptoms onset. The authors observed persistent positional down-beating and rotatory nystagmus with the fast phase beating towards the left ear both on the right head roll test and the right Dix-Hallpike test. Symptoms and signs disappeared in five days without any treatment. The authors 
proposed that nystagmus occurred because of light cupula of the right posterior canal. Head roll test and Dix-Hallpike test on the right side would cause the ampullopetal deflection of the right posterior canal cupula, provoking an inhibitory response with fast phases directed downward and towards the left ear. Either light debris attached to the cupula, such as blood cells ${ }^{16}$, or the increase of the density of the pericupular endolymph could cause light cupula ${ }^{17}$. Posterior canal light cupula would be rarer than lateral canal light cupula because light debris drops off easier into the ampulla of the lateral canal, which is in a higher position than the ampulla of the posterior canal.

Recently, Kim proposed another possible mechanism of light cupula caused by the difference in density between perilymph and endolymph. "When the density of perilymph becomes higher than that of endolymph by any cause, the membranous canal containing endolymph becomes buoyant within the perilymphatic space under the constant influence of gravity" 22.

Apogeotropic posterior canal BPPV is considered the most frequent posterior canal BPPV variant. It is also defined as reversed posterior canal BPPV because of the downbeat and torsional nystagmus towards the upward ear ${ }^{2,3,13}$. The two most accredited hypotheses are either otoconia in the non-ampullary arm of the canal or otoconia dislodged in the short arm, in the utricular side of the cupula.

In a recent multicentre study through v-HIT, Castellucci et al. ${ }^{23}$ demonstrated a decrease of the VOR gain of the affected canal in $72.9 \%$ of 59 cases of PDN, either from APC or from AC, increasing up to $88.6 \%$ in the subgroup with persistent down beat nystagmus, either positional or spontaneous. This datum was useful to distinguish between APC and contralateral AC. The authors suggested a partial jam of the affected canal as the mechanism of the disease. A partial canal jam might determine impaired responses for high-frequency stimuli, such as head impulses in vHIT, whereas low-frequency stimuli, such as the cupular stimulus of the endolymph flow activated during the positional test, remain active (low-pass filter). Data reported by Castellucci et al. ${ }^{23}$ highlight the difference between APC and TPC, in which the posterior canal VOR gain is not affected ${ }^{24}$.

As we pointed out in a previous paper ${ }^{13}$, only the conversion in TPC leads us to diagnose a definite APC. In that study, we analysed the oculomotor patterns of peripheral PDN and observed that APC was more common than AC. Our current clinical confirmed these data: APC represents a rare but not exceptional observation $-5 \%$ of all posterior canal BPPV - and it is much more frequent than $\mathrm{AC}-25$ vs 4 cases (ratio $\mathrm{APC} / \mathrm{AC}=6.25$ ).

We observed the conversion of APC in TPC in $14 / 25$ cas- es: in $8 / 14$ cases $(57.1 \%)$ after a liberatory manoeuver; in $6 / 14$ cases $(42.9 \%)$ suddenly and intensely during the prolonged maintenance of a forced head hyperextension position, sometimes without any dynamic movement applied to patients' heads, sometimes after a facilitating technique (see above). Both the conversion in TPC and the direct resolution through a liberatory manoeuver are compatible with otoconia dislodged in the non-ampullary arm of the affected posterior semicircular canal. Instead, the critical conversion through a prolonged still position does not appear compatible with non-ampullary arm canalolithiasis. In fact, the sudden fall of otoconia from this zone would provoke an ampullopetal endolymphatic flow, therefore evoking a downbeat and torsional apogeotropic nystagmus once again. The observed nystagmus can only be justified by an ampullofugal movement of otoconia from the periampullary zone to the most declivous part of the canal (Ewald's II law). A putative anomalous slope of the corresponding canal tract would initially cause an ampullopetal endolymphatic flow in the diagnostic position (Fig. 2B). At some point, either the prolonged maintenance of a forced head hyperextension position or the facilitating technique described above (Fig. 2C) could lead otoconia to move towards the area of a canal counter-slope, determining their ampullofugal sliding (Fig. 2D). Theoretically, nystagmus could also be caused by the exit of otoconia from the posterior canal ("liberatory" nystagmus). This circumstance was denied because TPC nystagmus was still present at the immediate retest by the Dix-Hallpike positioning: this means that otoconia, were still dislodged in the affected canal.

The observed nystagmus suggested the existence of at least two subgroups of APC: 1 . otoconia are in the non-ampul-

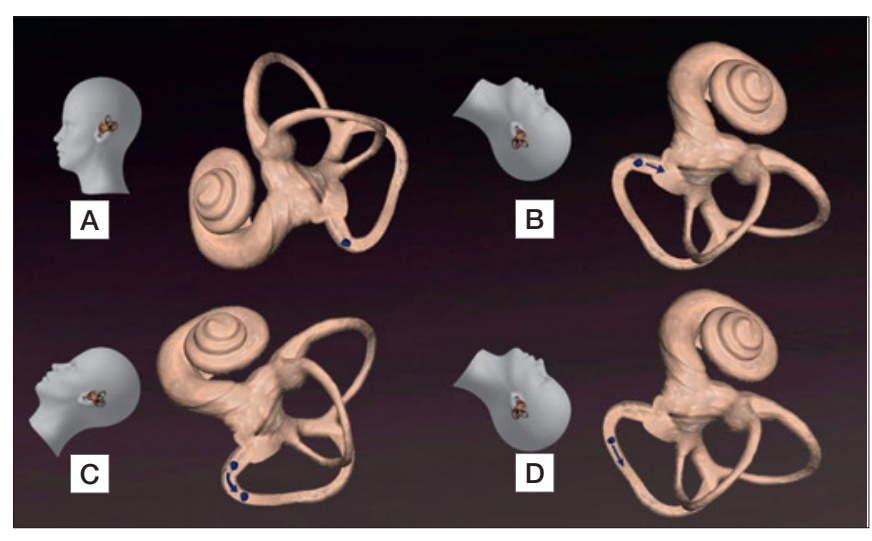

Figure 2. Our new model for some forms of apogeotropic posterior canal BPPV. Otoconia (blue circle) are in the periampullary tract of the posterior canal (A). During the Dix-Hallpike test they move ampullopetally (B). A quick and limited bowing of the head (C) followed by head hyperextension cause otoconia to move ampullophugally as in typical posterior canal BPPV (D). 
lary arm; 2. otoconia are in the periampullary zone of the canal.

During the diagnostic phase, in both cases otoconia movements provoke an ampullopetal, inhibitory endolymphatic flow, but only the periampullary position of otoconia could explain the sudden appearance of TPC nystagmus through a prolonged head hyperextension position. Otoconia in the non-ampullary tract, near the common crus, but also otoconia on the utricular side of the cupula, which could explain, in some of our cases, the direct resolution of canalolithiasis through liberatory manoeuvers.

Compared to the position stated in our previous article ${ }^{13}$, we are in fact currently more inclined to consider Büki 's model ${ }^{5,6}$ to explain some of the cases with the direct resolution of APC ("probable" grade). In our opinion, this possibility, however, is more plausible for long-lasting, untreated or not successfully treated cases.

A slight and transitory downbeat apogeotropic positional nystagmus is sometimes observed immediately after the treatment of TPC. It is probably determined by otoconial debris still present in the extreme distal portion of the canal ${ }^{25}$. This observation does not configure an autonomous variant, but, instead, indirectly confirms the hypothesis that in APC otoconia could be dislodged in the non-ampullary tract of the canal.

Scocco ${ }^{7}$ considered the periampullary position of otoconia in his variant, as well. He hypothesised canal stenosis of the distal periampullary tract and/or large-sized debris which allow otoconia to move ampullophugally from the periampullary zone to the stenosis not through the Dix-Hallpike test but returning to the sitting position. In our current series, we observed 5 similar cases. Our approach and their clinical evolution were the same that Scocco reported. Therefore, we agree with his model, which fully respects Ewald's laws.

The model proposed by Yetiser ${ }^{12}$ - non ampullary arm canalolithiasis to explain a demi-reversed form of left posterior canal BPPV - does not justify the dichotomy between the inversion of the torsional component, which was apogeotropic rather than geotropic, and the persistence of the vertical upbeat component. In fact, the apogeotropic direction of the torsional component is compatible with an ampullopetal flow, whereas the upbeat nystagmus is compatible with an ampullofugal flow. Another critical point is that the intensity of the nystagmus is stronger in its inhibitory phase than in the excitatory one. Both points contrast with Ewald's laws.

In our series, we observed two similar cases, characterised by inversion of the torsional phase of the nystagmus for the positioning side. In both cases, our conclusions were different from Yetiser's interpretation: after repeated positioning movements and liberatory manoeuvers, we observed the conversion in TPC of the contralateral posterior canal, in theory not examined through the positioning test on the other side. Could the different orientation of the affected semicircular canal, that would make it more susceptible to stimulation in contralateral positioning, be the hypothetical anomaly causing this variant? It could, but it should be noted that, in both our cases, the affected canal was activated on its proper side positioning, causing the disappearance of a nearly anecdotal variant!

"Subjective BPPV" and "trunk retropulsion in sitting up without observable nystagmus" represented a not rare possible evolution of non treated cases because of either the delayed diagnosis or a not entirely resolutive therapy. The response to physical therapy was good.

\section{Conclusions}

The interpretative schematism of posterior canal BPPV and, in particular, the idea that all cases of positional downbeat nystagmus are due to central neurological diseases, have been lost ${ }^{19,20,21,26}$.

Less common subtypes of BPPV, hypothetically due to a non-standard position of otoconia in the affected canal, or canal anomalies, or partial canal jam, are possible. Their interpretations are suitable only if they agree with Ewald's laws: if they do, the observed nystagmus pattern has to be considered compatible with peripheral mechanisms. Therefore, in a less demanding way, the less common posterior canal BPPV types must be considered as "variant forms" and no longer defined as "atypical forms".

In the spirit of what Epley wrote ${ }^{16}$, the more different a nystagmus is from the most common nystagmus observed in that disease, the more likely other diseases could be its cause. The less common variants of posterior canal BPPV require an in-depth clinical study to detect any signs of cerebellar diseases. Careful neurotological examination may limit the need for brain imaging to cases not converted into a typical form and not resolved through the usual physical therapy, but this is achievable only if the knowledge of the various possible types of posterior canal BPPV is an important part of the cultural heritage of all otoneurologists.

\section{Acknowledgements}

We thank Architect Genoveffa Errico for the original illustrations.

\section{References}

1 von Brevern M, Bertholon P, Brandt T, et al. Benign paroxysmal positional vertigo: diagnostic criteria. J Vestib Res 2015;25:105-117. https://doi.org/10.3233/VES-150553 
2 Agus G, Puxeddu R, Demontis GP, et al. Atypical "reversed" paroxysmal positioning nystagmus in benign paroxysmal positional vertigo. Acta Otolaryngol Suppl 1995;520 Pt 1:143-147. https://doi. org/10.3109/00016489509125213

3 Vannucchi P, Pecci R, Giannoni B. Posterior semicircular canal benign paroxysmal positional vertigo presenting with torsional downbeating nystagmus: an apogeotropic variant. Int J Otolaryngol 2012;2012:413603. https://doi.org/10.1155/2012/413603

4 Oas JG. Benign paroxysmal positional vertigo. A clinician's perspective. Ann NY Acad Sci 2001;942:201-209 https://doi. org/10.1111/j.1749-6632.2001.tb03746.x

5 Büki B, Simon L, Garab S, et al. Sitting-up vertigo and trunk retropulsion in patients with benign positional vertigo but without positional nystagmus. J Neurol Neurosurg Psychiatry 2011;82:98-104. https:// doi.org/10.1136/jnnp.2009.199208

6 Büki B. Benign paroxysmal positional vertigo-toward new definitions. Otol Neurotol 2014;35:323-328. https://doi.org/10.1097/ MAO.0000000000000197

7 Scocco DH, Garcia IE, Barreiro MA. Sitting up vertigo. proposed variant of posterior canal benign paroxysmal positional vertigo. Otol Neurotol 2019;40:497-503. https://doi.org/10.1097/ MAO.0000000000002157

8 Asprella Libonati G. Benign paroxysmal positional vertigo and positional vertigo variants. Int J Otorhinolaryngol Clin 2012;4:25-40. https://doi.org/10.5005/jp-journals-10003-1085

9 Tomanovic T, Büki B. The diagnostic framework of peripheral positional vertigo and dizziness (PPVD): a new concept based on the observation of alcolohol-induced posterior canal light cupula. Acta Otolaryngol Case Rep 2016;1:40-48. https://doi.org/10.1080/237724 84.2016.1209640

10 Ichimura A, Otsuka K. Persistent down-beating torsional positional nystagmus: posterior semicircular canal light cupula? Case Rep Otolaryngol 2016;2016:1249325. https://doi.org/10.1155/2016/1249325

11 Hiruma K, Numata T. Positional down beating nystagmus showing neutral points. ORL J Otorhinolaryngol Relat Spec 2004;66:46-50. https://doi.org/10.1159/000077234

12 Yetiser S. A new variant of posterior canal benign paroxysmal positional vertigo: a non ampullary or common crus canalolithiasis. Case Rep Otolaryngol 2015;2015:816081. https://doi. org/10.1155/2015/816081

13 Califano L, Salafia F, Mazzone S, et al. Anterior canal BPPV and apogeotropic posterior canal BPPV: two rare forms of vertical canalolithiasis. Acta Othorinolaryngol Ital 2014;34:189-197.

14 Califano L, Capparuccia PGG, Di Maria D, et al. Treatment of benign paroxysmal positional vertigo of posterior semicircular canal by "quick liberatory rotation manoeuvre". Acta Otorhinolaryngol Ital 2003;23:161-167.
15 Vannucchi P, Pecci R, Giannoni B, et al. Apogeotropic posterior semicircular canal benign paroxysmal positional vertigo: some clinical and therapeutic considerations. Audiol Res 2015;5:130. https://doi. org/10.4081/audiores.2015.130

16 Epley JM. Positional vertigo related to semicircular canalithiasis. Otolaryngol Head Neck Surg 1995;112:154-161. https://doi.org/10.1016/ S0194-59989570315-2

17 Schratzenstaller B, Wagner-Manslaub C, Alexiou C, et al. High-resolution three-dimensional magnetic resonance imaging of the vestibular labyrinth in patients with atypical and intractable benign positional vertigo. ORL 2001;63:165-177. https://doi.org/ 10.1159/000055734

18 Tang X, Tang J, Gong L, et al. Adverse effects of semicircular circle angles variation on Epley repositioning procedure: a study on reconstruction of Micro-CT images 3D. Acta Otolaryngol 2020;140:99104. https://doi.org/10.1080/00016489.2019.1699953

19 Bertholon P, Bronstein AM, Davies RA, et al. Positional down beating nystagmus in 50 patients: cerebellar disorders and possible anterior semicircular canalithiasis. J Neurol Neurosurg Psychiatry 2002;72:366-372. 10. https://doi.org/1136/jnnp.72.3.366

20 ChoiJY,KimJH,KimHJ, etal.Central paroxysmalpositional nystagmus: characteristics and possible mechanisms. Neurology 2015;84:22382246. https://doi.org/10.1212/WNL.0000000000001640

21 De Schutter E, Adham ZO, Kattah JC. Central positional vertigo: a clinical-imaging study. Prog Brain Res 2019;249:345-360. https://doi. org/10.1016/bs.pbr.2019.04.022

22 Kim CH, Pham NC. Density difference between perilymph and endolymph: a new hypothesis for light cupula phenomenon. Med Hypotheses 2019;123:55-59. https://doi.org/10.1016/j.mehy.2018.12.017

23 Castellucci A, Malara P, Martellucci, et al. Feasibility of using the video-head impulse test to detect the involved canal in benign paroxysmal positional vertigo presenting with positional downbeat nystagmus. Front Neurol 2020;11:578588. https://doi.org/10.3389/ fneur.2020.578588

24 Califano L, Iannella R, Mazzone S, et al. The Video Head Impulse Test in the acute stage of posterior canal benign paroxysmal positional vertigo. Acta Otorhinolaryngol Ital 2021;41:69-76. https://doi. org/10.14639/0392-100X-N1033

25 Oh EH, Lee J-H, Kim H-J, et al. Incidence and clinical significance of positional downbeat nystagmus in posterior canal benign paroxysmal positional vertigo. J Clin Neurol 2019;15:143-148. https://doi. org/10.3988/jen.2019.15.2.143

26 Cambi J, Astore S, Mandalà $\mathrm{M}$, et al. Natural course of positional down-beating nystagmus of peripheral origin. J Neurol 2013;260:1489-1496. https://doi.org/10.1007/s00415-012-6815-9 